\title{
Neoadjuvant chemotherapy for atypical teratoid rhabdoid tumors: case report
}

\author{
Meena Thatikunta, MD, ${ }^{1}$ lan Mutchnick, MD, ${ }^{1-3}$ Jennifer Elster, MD, ${ }^{4,5}$ \\ Matthew P. Thompson, DO, MS, ${ }^{6}$ Michael A. Huang, MD, ${ }^{7}$ Aaron C. Spalding, MD, PhD, ${ }^{8}$ and \\ Thomas Moriarty, MD, $\mathrm{PhD}^{1-3}$
}

\begin{abstract}
'Department of Neurosurgery, University of Louisville School of Medicine; '2Pediatric Neurosurgery, Norton Children's Hospital; ${ }^{3}$ Norton Neuroscience Institute, Louisville, Kentucky; ${ }^{4}$ Pediatric Hematology/Oncology, UC San Diego School of Medicine; ${ }^{5}$ Rady Children's Hospital-San Diego, California; ${ }^{6}$ Pediatric and Perinatal Pathology Associates, Norton Children's Hospital; ${ }^{7}$ Pediatric Cancer \& Blood Disorders, University of Louisville School of Medicine; and ${ }^{8}$ Radiation Oncology, Norton Cancer Institute, Norton Healthcare, Louisville, Kentucky
\end{abstract}

\begin{abstract}
Atypical teratoid rhabdoid tumors (ATRTs) are a rare pediatric brain tumor with high mortality rate. Several large series have reported achieving gross-total resection (GTR) in less than $50 \%$ of patients due to the lesions' large size, vascularity, and limited blood volume in young patients. While neoadjuvant chemotherapy for choroid plexus carcinomas in pediatric patients has become widely accepted, it has not been used as widely for other pediatric brain tumors. To the best of the authors' knowledge, there are only 3 published cases of neoadjuvant chemotherapy for ATRTs. In the present report, the authors present a fourth case of neoadjuvant chemotherapy for ATRT and review the available literature on this strategy. A 17-month-old child presented with a left ventricular ATRT for which imaging raised concern for a highly vascularized tumor. The authors undertook neoadjuvant chemotherapy with 2 cycles of Head Start II therapy, which reduced the size of the ventricular tumor by $35 \%$ and decreased the vascularity of the lesion on imaging. The estimated blood loss during resection was $425 \mathrm{ml}$ and GTR was achieved. The patient continued with postoperative chemotherapy but suffered an on-therapy recurrence. While higher-quality data are necessary, available evidence suggests that neoadjuvant chemotherapy can reduce the size and vascularity of ATRTs and facilitate a surgical avenue for large or "inoperable" tumors.
\end{abstract}

https://thejns.org/doi/abs/10.3171/2016.12.PEDS16427

KEY WORDS ATRT; atypical teratoid rhabdoid tumor; neoadjuvant chemotherapy; pediatric brain tumor; gross-total resection; Head Start; choroid plexus carcinoma; oncology

$\Lambda$ TYPICAL teratoid rhabdoid tumors (ATRTs) of the CNS are a rare and aggressive pediatric tumor, and the mean survival time ranges from 6 to 11 months after diagnosis., ${ }^{43,15}$ Young children are disproportionately affected: $66 \%$ of cases occur in children younger than 2 years. ${ }^{4,9,14,15,18,22} \mathrm{Few}$ data are available for ATRTs and no standard therapy exists. Gardner et al. ${ }^{6}$ reported on outcomes of 13 patients with CNS ATRTs treated using the Head Start I and II protocols consisting of intensive induction chemotherapy (vincristine, cisplatin, etoposide, and cyclophosphamide) followed by high-dose chemotherapy with autologous hematopoietic stem cell rescue. The Head Start II regimen differs only in the addition of high-dose methotrexate. In series reported by Gardner et al., 6 of 13 patients had a complete response to induction chemotherapy. Three of the 7 being treated with the Head Start II protocol were alive without evidence of disease more than 3 years after therapy ended. Importantly, all 3 of these patients had gross-total resection (GTR) or near-total resection (NTR). ${ }^{6}$ However, ATRTs can present a significant challenge to the surgeon seeking GTR or NTR. Vascularity, tumor size, and small total-body blood volumes can significantly limit resectability of these tumors. Several large series have reported GTR in less than $50 \%$ of patients, but GTR strongly predicts outcome. ${ }^{3,5,8,9,12,13,20}$

Neoadjuvant chemotherapy has been used for pediatric choroid plexus carcinomas (CPCs) since the $1990 \mathrm{~s}^{11,17}$, ${ }^{21,23}$ and is becoming the standard of care for CPCs, which similarly afflict young patients with limited blood volume,

ABBREVIATIONS ATRT = atypical teratoid rhabdoid tumor; CPC = choroid plexus carcinoma; EBL/EBV = estimated blood loss/estimated blood volume; EVD = external ventricular drain; GTR = gross-total resection; NTR = near-total resection; SIADH = syndrome of inappropriate antidiuretic hormone.

SUBMITTED July 24, 2016. ACCEPTED December 6, 2016.

INCLUDE WHEN CITING Published online March 10, 2017; DOI: 10.3171/2016.12.PEDS16427. 
grow to a large size, and have plentiful vascular supply from the choroidal vessels. ${ }^{16,19,24}$ In 2015, Schneider et al. ${ }^{19}$ used neoadjuvant chemotherapy to treat CPCs and reported that $72 \%$ of patients receiving neoadjuvant chemotherapy experienced a GTR compared with $26 \%$ of those not receiving neoadjuvant chemotherapy $(\mathrm{p}=0.02)$. In a logistic regression analysis, only neoadjuvant chemotherapy was an independent predictor of intraoperative blood loss. ${ }^{19}$ Pathological examination of specimens from second-look surgeries reveal tumoral fibrosis and collagenization particularly around blood vessels and tumor nests. ${ }^{11} h S N F 5 / I N I-I$ gene inactivation, previously thought to be diagnostic for ATRT, has been implicated in CPCs. Thus, CPCs may be similar in tumorigenesis to ATRTs. ${ }^{7}$

In case series reported by Iwama et al. and Van Poppel et al., there were 3 ATRT patients who underwent neoadjuvant chemotherapy. ${ }^{10,25}$ The first patient was diagnosed at 2 months of age with a 70-mm-in-largest-diameter ventricular ATRT. He underwent 4 rounds of chemotherapy, which decreased the size of the tumor. His estimated blood loss/estimated blood volume (EBL/EBV) was 39\%, and the patient received a blood transfusion intraoperatively. GTR was achieved..$^{10}$ The second patient was diagnosed at 20 months of age, underwent subtotal resection, and then underwent 4 rounds of induction chemotherapy. Intraoperatively, blood loss was minimal at 5.4\% EBL/EBV and GTR was achieved. The third patient was diagnosed at 25 months of age, underwent biopsy, and GTR was achieved after 3 courses of induction chemotherapy. Intraoperatively, blood loss was $27.7 \% \mathrm{EBL} / \mathrm{EBV} .{ }^{25}$ The present case is, to the best of our knowledge, the fourth reported case in which neoadjuvant chemotherapy was used to treat an ATRT. Local IRB approval was obtained prior to submission of this paper for publication.

\section{Case Report}

Presentation and Examination

A 17-month-old boy without significant birth or medical history, presented with a 2-week history of emesis occurring 2 to 3 times per day and more consistently in the morning. In the preceding days, he had become more irritable, with poor oral intake and a demonstrated unwillingness to walk. He had multiple emergency department visits in the preceding 3 months but did not exhibit any focal neurological deficit and so had not undergone cranial imaging. At presentation, he was sleepy but easily arousable, playful, and interactive. He had a nonfocal neurological examination, although his mother reported a significant decrease in his right-sided motor function compared with the left. He was without pupillary abnormality, and his anterior fontanelle was soft and flat. MRI of the brain demonstrated a large left ventricular tumor originating from the lateral aspect of the thalamus, thought likely to be an ATRT rather than a primitive neuroectodermal tumor (PNET) or CPC causing obstructive hydrocephalus. There was diffuse, thickened enhancement of the lining of the left lateral ventricle. MRI of the spine demonstrated diffuse lumbosacral metastases. While in the hospital, a significant left-sided dominance with delayed initiation of right upper-extremity movement became apparent. Mild

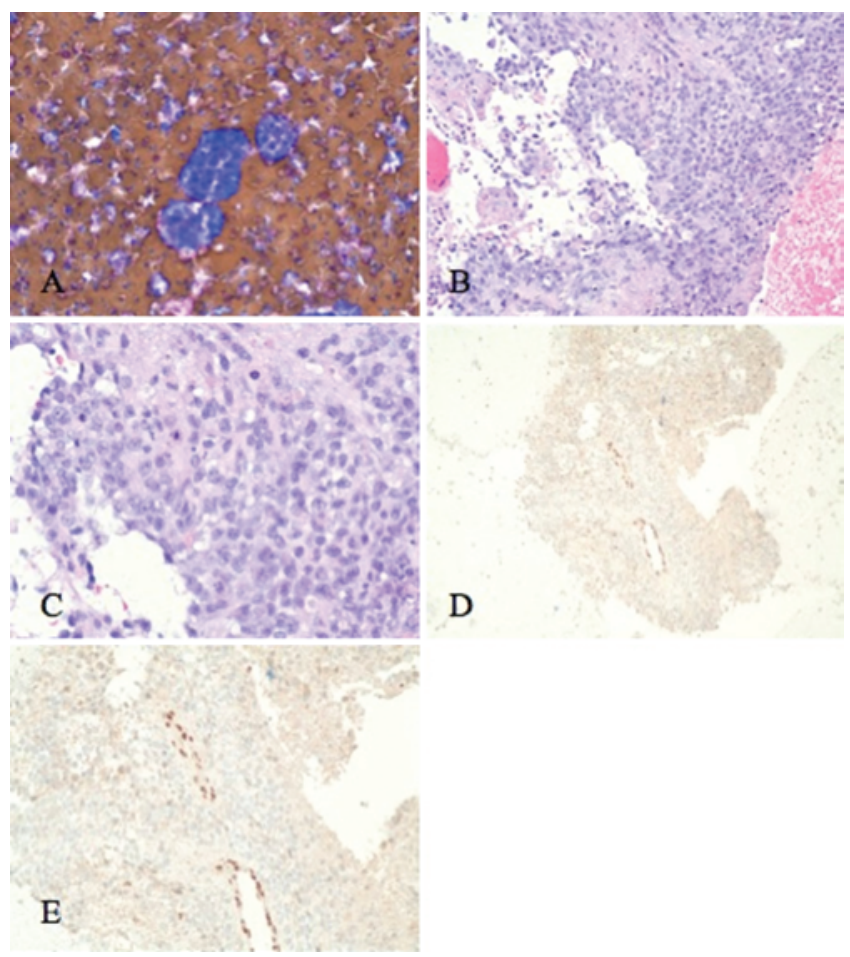

FIG. 1. A: CSF cytology showing diffuse clumps of large malignant-appearing cells. Original magnification $\times 10$. B and C: $\mathrm{H} \& \mathrm{E}$-stained sections of the cell block demonstrating large cells with minimal cytoplasm and irregular nuclei. Original magnification $\times 10$ (B) and $\times 20$ (C). D and $\mathrm{E}$ : Immunostains for INI-1 were negative in the tumor cells (positive in the internal control endothelium). Original magnification $\times 10$ (D) and $\times 20$ (E). Figure is available in color online only.

syndrome of inappropriate antidiuretic hormone (SIADH) was also discovered.

\section{Initial Management}

The patient underwent placement of a bilateral external ventricular drain (EVD) for obstructive hydrocephalus occluding the foramen of Monro. CSF obtained from the initial EVD placement was sent for cytological examination and the finding confirmed the diagnosis of CNS ATRT. The patient was not considered a surgical candidate due to widespread metastases and extensive vascularity of the tumor. His parents elected to pursue chemotherapy with an intention to proceed with resection if the tumor responded sufficiently. Internal CSF diversion was required in anticipation of cytotoxic chemotherapy, and the patient underwent insertion of both a right-sided ventriculoperitoneal shunt with Strata valve set at a performance level of 2.5 and a left-sided Ommaya reservoir to keep the left lateral ventricle open for future surgery. Figure 1 shows the final pathology on the CSF sample, demonstrating a highly cellular specimen composed of clumps of large cells with large and irregular nuclei and minimal cytoplasm. The cells were negative for AE1/AE3, GFAP, synaptophysin, OCT-4, AFP, CAM5.2, and INI-1 on immunohistochemistry.

\section{Chemotherapy}

After discussion with the family regarding the poor 

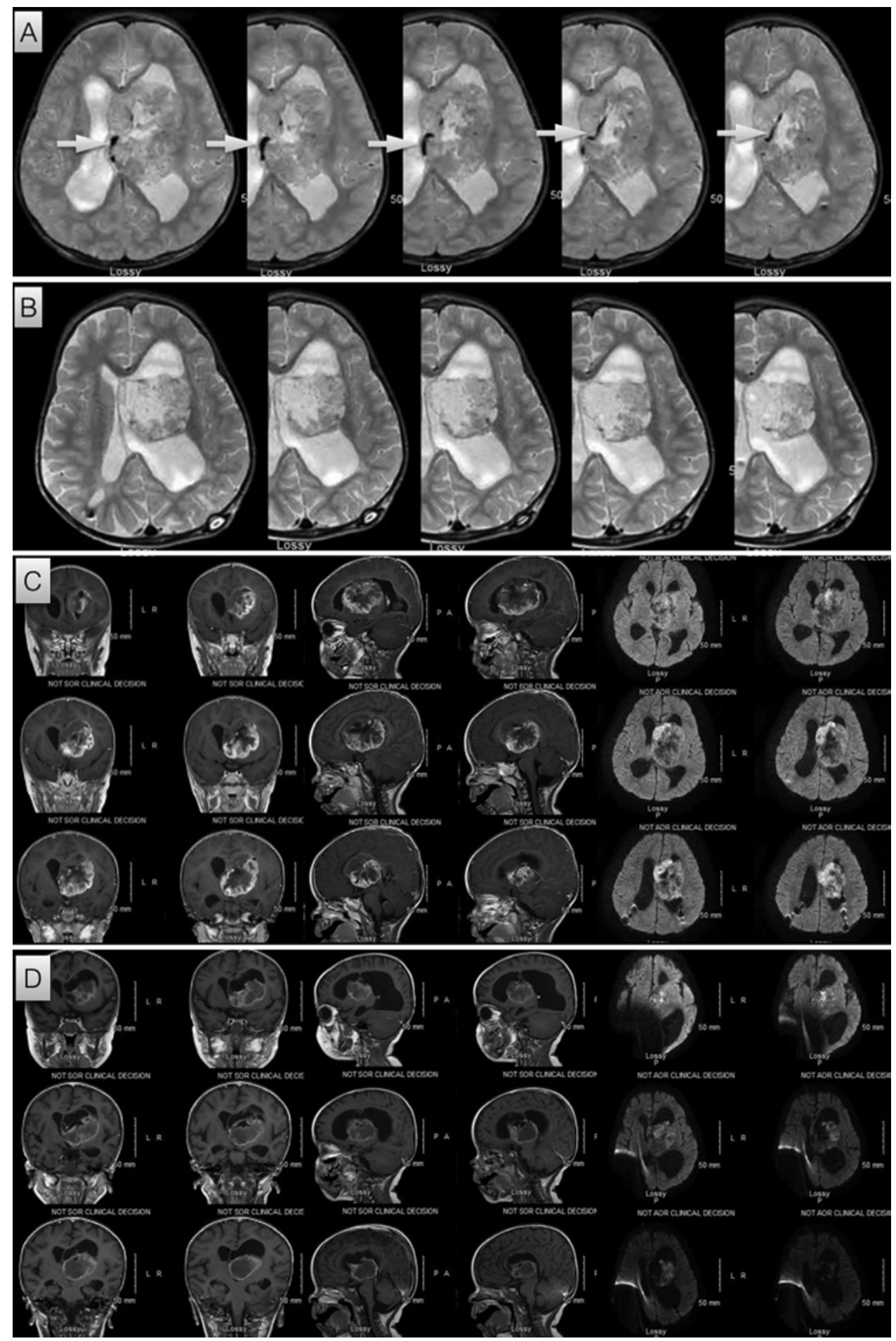

FIG. 2. Vascular changes were evident on the axial 3D T2-weighted images. Before neoadjuvant chemotherapy note the large medial vascular structure indicated by the arrows (A). After neoadjuvant chemotherapy the medial vascular structure was no longer seen and there was a loss of cellularity (B). Tumor changes were also significant in sagittal and coronal postcontrast and diffusion-weighted images. Before neoadjuvant chemotherapy (C) versus after neoadjuvant chemotherapy (D), there was a $35 \%$ decrease in volumetrically determined size and loss of contrast enhancement (D). 


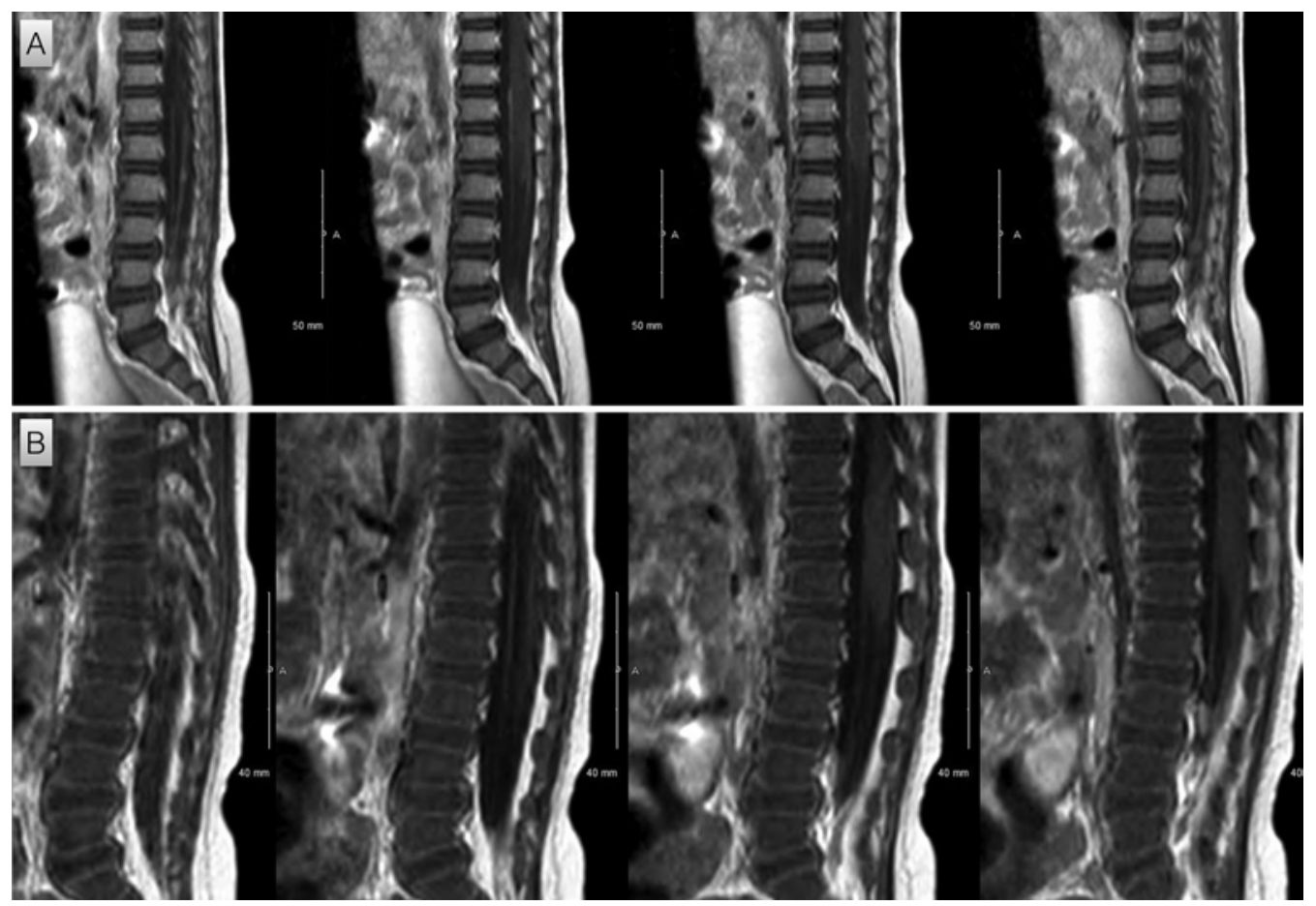

FIG. 3. Sagittal postcontrast T1-weighted MR images of the lumbar spine before and after neoadjuvant chemotherapy. Multiple small nodules were present in the lumbosacral thecal sac consistent with spinal leptomeningeal carcinomatosis (A). Interval resolution of diffuse spinal leptomeningeal carcinomatosis. Note the diffuse osseous posttreatment effect (B).

prognosis and the available options, chemotherapy was started as per the induction regimen of Head Start II. The patient received 2 cycles of chemotherapy consisting of vincristine, cisplatin, etoposide, cyclophosphamide, and high-dose methotrexate. After the first cycle, autologous hematopoietic stem cells were collected in anticipation of treatment consolidation with high-dose chemotherapy and autologous hematopoietic stem cell rescue if an eventual GTR was achieved. Resolution of spinal metastases was seen after 2 rounds of chemotherapy. Chemotherapy was complicated by significant mucositis requiring opiate management and nasojejunal tube placement for nutritional support. The patient required multiple transfusions and granulocyte-colony stimulating factor support for neutropenia. Following the second cycle of chemotherapy, he developed pneumonia that was treated for bacterial and presumed fungal causes. In addition to upper oral and pulmonary issues, the patient had a significant exacerbation of his underlying SIADH thought to be secondary to administration of vincristine. Despite these chemotherapeutic complications, the patient was able to complete 2 cycles of therapy.

\section{Operation}

Two months after presentation, following 2 cycles of chemotherapy, a repeat MRI brain scan showed 35\% reduction in tumor size as determined by the reviewing oncology team together with the pediatric neuroradiologist. 3D T2-weighted axial MR images in Fig. 2 show the significant near disappearance of large medially draining veins, and the MR images in Fig. 3 show resolution of lumbosacral leptomeningeal disease.

The patient's mother elected to proceed with surgical treatment of her son. A left pterional craniotomy was performed and frameless stereotactic navigation was employed. Most of the resection was straightforward due to the large extent of avascular, necrotic tissue, likely induced by neoadjuvant chemotherapy. However, there was marked increase in vascularity and bleeding at the margins, the medial margin in particular. Because a GTR in this context was so valuable and the patient had had such a robust response to neoadjuvant chemotherapy, aggressive resection was pursued and achieved. The patient experienced $425 \mathrm{ml}$ of blood loss, a 52\% EBL/EBV, and he was transfused with $40 \mathrm{ml} / \mathrm{kg}$ packed red blood cells and $2 \mathrm{~L}$ of crystalloid fluid. At this point, hemostasis was achieved, and the operation was ended; a left frontal EVD was placed before closing. Intraoperatively, the patient experienced hyperkalemia (likely secondary to transfusion) with peak potassium levels of $7.6 \mathrm{mEq} / \mathrm{L}$ and peaked $\mathrm{T}$ waves on electrocardiography, easily managed with insulin and glucose replacement.

Figure 4 shows tumor pathology; $80 \%$ of the specimen sent to pathology was necrotic tumor with the remaining viable tumor cells showing irregular nuclei, prominent nucleoli, and occasional classic "rhabdoid" cytoplasm. Hemosiderin deposition and reactive changes were found with foci of ependymal lining and periventricular brain with viable tumor cells invading beneath the ependymal epithelium that were BAF-47 negative and showed 40\%$50 \%$ positivity for Ki 67 . PHH3 staining in the areas of 


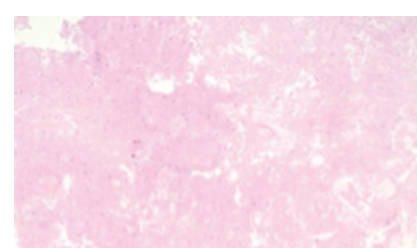

A

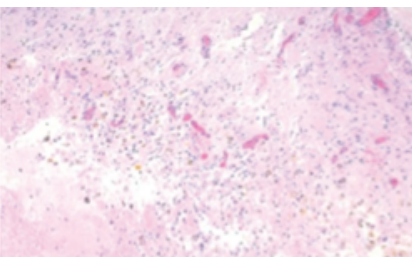

B
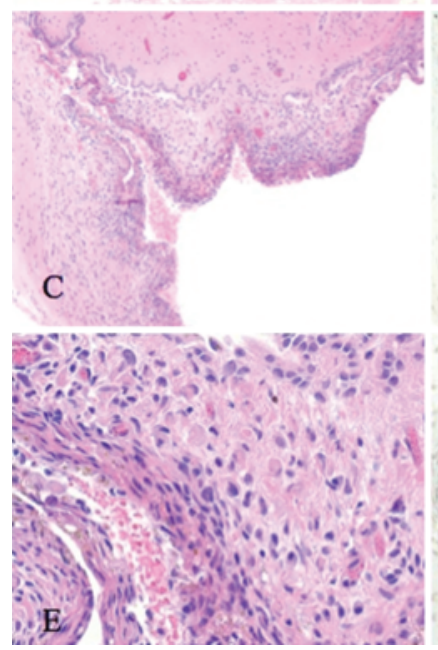

FIG. 4. A and B: The resected material was composed primarily of nonviable necrotic tumor and areas of treatment effect with hemosiderinladen macrophages. C-F: There were areas of viable subependymal tumor cells with a rhabdoid morphology and high Ki 67 labeling that are negative for INI-1. H \& E (A-C and E), INI-1 (D), and Ki 67 (F), original magnification $\times 10(A-D), \times 40(E)$, and $\times 20(F)$. Figure is available in color online only. viable tumor showed a range of $0-3$ mitotic figures per high-power field.

Immediately postoperatively, the patient was extubated on $3 \mathrm{~L}$ of oxygen via nasal cannula; however, during postoperative CT scanning, he developed increased secretions, tachypnea, and stridor for which he received epinephrine and was reintubated. Due to pulmonary issues (perhaps secondary to the 2 rounds of chemotherapy and the longer-term effects of severe mucositis), he remained intubated until postoperative Day 5. Neurologically, he moved his right upper-extremity well and his right lower-extremity moderately less than prior to surgery; during the subsequent months the patient's right lower-extremity weakness improved to baseline according to his mother. Figure 5 shows a GTR on his postoperative MRI.

\section{Postoperative Course}

The patient completed 3 postoperative cycles of induction chemotherapy for a total of 5 cycles of Head Start II therapy. The plan was to proceed to consolidation with high-dose chemotherapy and autologous hematopoietic stem cell rescue, but this therapy was delayed due to persistent respiratory viral infections. At restaging in preparation for proceeding with therapy, he was found to have radiographic evidence of thoracolumbar recurrence and frank CSF cytological positivity. The family decided not to pursue any further cytotoxic therapy. He received outpatient palliative care services and died at 27 months of age, having survived 10 months since diagnosis.

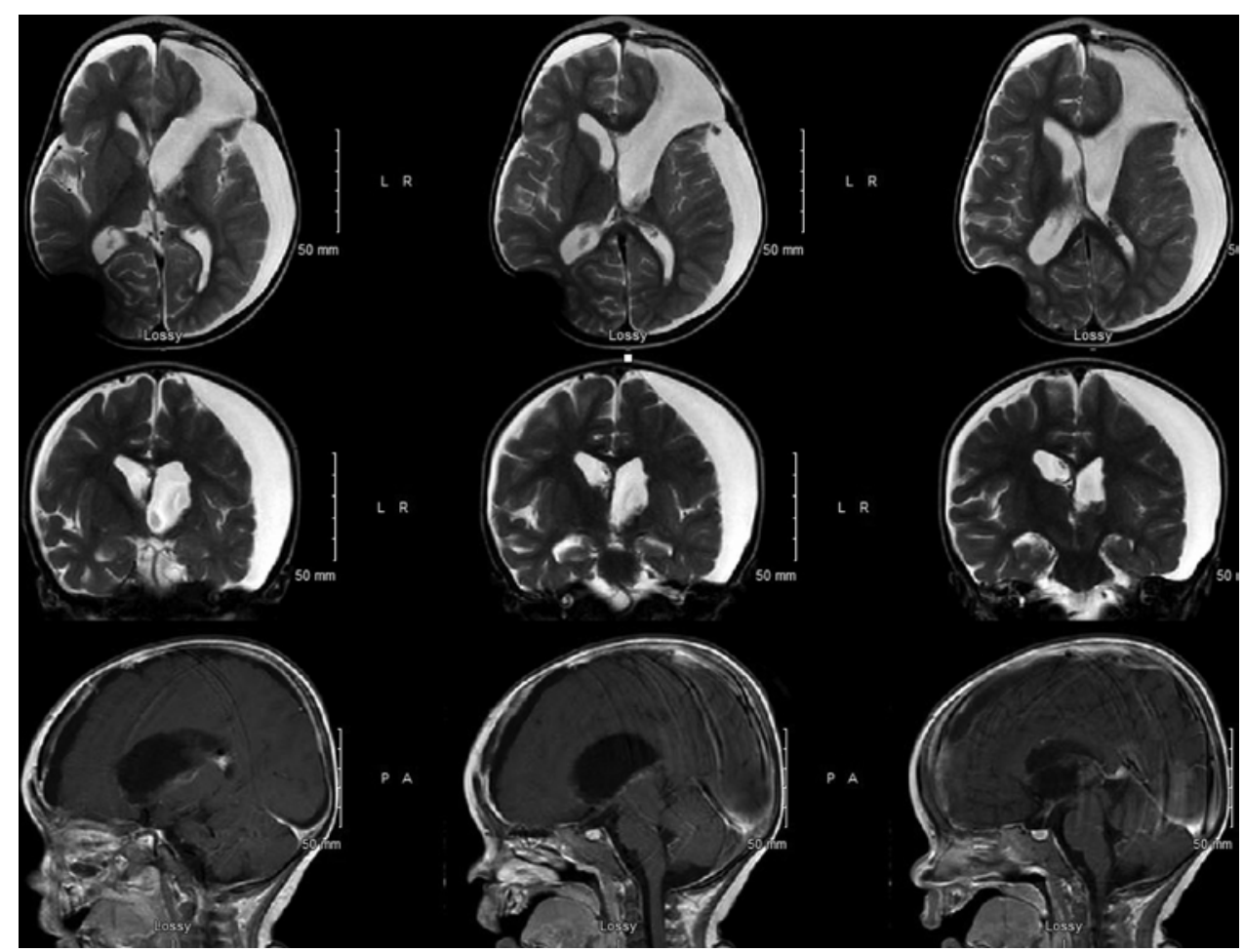

FIG. 5. Axial (upper) and coronal (center) T2-weighted and sagittal T1-weighted postcontrast (lower) MR images obtained 1 month postoperatively demonstrating GTR of an intraventricular ATRT in the child at 20 months of age. 


\section{Discussion}

The pediatric neurosurgeon can face significant challenges when managing pediatric brain tumors with their large sizes and vascular relationships in the context of smaller total blood volumes. There exists a growing body of data to support the use of neoadjuvant chemotherapy to treat such large, vascular tumors. ${ }^{10,25}$ Neoadjuvant chemotherapy appears to increase fibrosis of the tumor, creating a clearer surgical plane between pathological and normal brain parenchyma, as well as collagenization of blood vessels. ${ }^{11}$ Neoadjuvant chemotherapy has been shown to decrease intraoperative blood loss and tumor size.,10,11,19,21 While it is not feasible in emergency or urgent cases, neoadjuvant chemotherapy expands the armamentarium of the pediatric neurosurgeon to approach tumors that may have otherwise been deemed "inoperable." Future development of chemotherapeutic agents may be directed toward collagenization and fibrosis of tumoral blood supply for the specific purpose of enhancing surgical candidacy of patients with particularly vascular brain tumors.

In the present case, resection was complicated by the size of the tumor, its vascularity, and expense of total blood volume in this small child. Since these issues are common in vascular pediatric brain tumors, methods to increase the neurosurgeon's ability to achieve GTR safely are highly valuable. Patients presenting with ATRT metastases have a median survival of 3 months; 5 our patient presented at 17 months of age and died at 27 months of age. GTR is one of a few tools for ATRTs that demonstrably lengthens survival. ${ }^{8,12,13}$ The benefits of tumor reduction must be weighed against the increased risk of surgery in the context of neoadjuvant chemotherapy. SIADH, bacterial infections including sepsis and recurrent fungal infections, and mucositis are well-known toxicities among Head Start II patients. These toxicities can lead to challenging critical care scenarios and may lead to ultimate demise. ${ }^{2,6}$ In the report by Van Poppel et al., neoadjuvant chemotherapy was associated with tumor-specific complications including intratumoral hemorrhage and peritumoral edema, as well as chemotherapy-induced thrombocytopenia. ${ }^{25}$ Specifically with ATRTs, one neoadjuvant ATRT patient has died of sepsis, although it is unknown if this was attributed to the chemotherapy. ${ }^{10}$

Given relatively low rates for GTR reported in the literature and the importance of GTR in the future survival in ATRT patients, therapies enabling GTR are particularly valuable. Neoadjuvant chemotherapy may be considered in challenging ATRT cases.

\section{References}

1. Addo NK, Kamaly-Asl ID, Josan VA, Kelsey AM, Estlin EJ: Preoperative vincristine for an inoperable choroid plexus papilloma: a case discussion and review of the literature. $\mathbf{J}$ Neurosurg Pediatr 8:149-153, 2011

2. Altshuler C, Haley K, Dhall G, Vasquez L, Gardner SL, Stanek J, et al: Decreased morbidity and mortality of autologous hematopoietic transplants for children with malignant central nervous system tumors: the 'Head Start' trials, 1991-2009. Bone Marrow Transplant 51:945-948, 2016

3. Athale UH, Duckworth J, Odame I, Barr R: Childhood atypical teratoid rhabdoid tumor of the central nervous system: a meta-analysis of observational studies. J Pediatr Hematol Oncol 31:651-663, 2009

4. Burger PC, Yu IT, Tihan T, Friedman HS, Strother DR, Kepner JL, et al: Atypical teratoid/rhabdoid tumor of the central nervous system: a highly malignant tumor of infancy and childhood frequently mistaken for medulloblastoma: a Pediatric Oncology Group study. Am J Surg Pathol 22:1083-1092, 1998

5. Buscariollo DL, Park HS, Roberts KB, Yu JB: Survival outcomes in atypical teratoid rhabdoid tumor for patients undergoing radiotherapy in a Surveillance, Epidemiology, and End Results analysis. Cancer 118:4212-4219, 2012

6. Gardner SL, Asgharzadeh S, Green A, Horn B, McCowage $\mathrm{G}$, Finlay J: Intensive induction chemotherapy followed by high dose chemotherapy with autologous hematopoietic progenitor cell rescue in young children newly diagnosed with central nervous system atypical teratoid rhabdoid tumors. Pediatr Blood Cancer 51:235-240, 2008

7. Gessi M, Giangaspero F, Pietsch T: Atypical teratoid/ rhabdoid tumors and choroid plexus tumors: when genetics "surprise" pathology. Brain Pathol 13:409-414, 2003

8. Ginn KF, Gajjar A: Atypical teratoid rhabdoid tumor: current therapy and future directions. Front Oncol 2:114, 2012

9. Hilden JM, Meerbaum S, Burger P, Finlay J, Janss A, Scheithauer BW, et al: Central nervous system atypical teratoid/rhabdoid tumor: results of therapy in children enrolled in a registry. J Clin Oncol 22:2877-2884, 2004

10. Iwama J, Ogiwara H, Kiyotani C, Terashima K, Matsuoka $\mathrm{K}$, Iwafuchi $\mathrm{H}$, et al: Neoadjuvant chemotherapy for brain tumors in infants and young children. J Neurosurg Pediatr 15:488-492, 2015

11. Lafay-Cousin L, Mabbott DJ, Halliday W, Taylor MD, Tabori U, Kamaly-Asl ID, et al: Use of ifosfamide, carboplatin, and etoposide chemotherapy in choroid plexus carcinoma. J Neurosurg Pediatr 5:615-621, 2010

12. Meyers SP, Khademian ZP, Biegel JA, Chuang SH, Korones DN, Zimmerman RA: Primary intracranial atypical teratoid/ rhabdoid tumors of infancy and childhood: MRI features and patient outcomes. AJNR Am J Neuroradiol 27:962-971, 2006

13. Nicolaides T, Tihan T, Horn B, Biegel J, Prados M, Banerjee A: High-dose chemotherapy and autologous stem cell rescue for atypical teratoid/rhabdoid tumor of the central nervous system. J Neurooncol 98:117-123, 2010

14. Ostrom QT, Chen Y, M de Blank P, Ondracek A, Farah P, Gittleman H, et al: The descriptive epidemiology of atypical teratoid/rhabdoid tumors in the United States, 2001-2010. Neuro Oncol 16:1392-1399, 2014

15. Packer RJ, Biegel JA, Blaney S, Finlay J, Geyer JR, Heideman R, et al: Atypical teratoid/rhabdoid tumor of the central nervous system: report on workshop. J Pediatr Hematol Oncol 24:337-342, 2002

16. Passariello A, Tufano M, Spennato P, Quaglietta L, Verrico A, Migliorati R, et al: The role of chemotherapy and surgical removal in the treatment of choroid plexus carcinomas and atypical papillomas. Childs Nerv Syst 31:1079-1088, 2015

17. Razzaq AA, Cohen AR: Neoadjuvant chemotherapy for hypervascular malignant brain tumors of childhood. Pediatr Neurosurg 27:296-303, 1997

18. Rorke LB, Packer RJ, Biegel JA: Central nervous system atypical teratoid/rhabdoid tumors of infancy and childhood: definition of an entity. J Neurosurg 85:56-65, 1996

19. Schneider C, Kamaly-Asl I, Ramaswamy V, Lafay-Cousin L, Kulkarni AV, Rutka JT, et al: Neoadjuvant chemotherapy reduces blood loss during the resection of pediatric choroid plexus carcinomas. J Neurosurg Pediatr 16:126-133, 2015

20. Schrey D, Carceller Lechón F, Malietzis G, Moreno L, 
Dufour C, Chi S, et al: Multimodal therapy in children and adolescents with newly diagnosed atypical teratoid rhabdoid tumor: individual pooled data analysis and review of the literature. J Neurooncol 126:81-90, 2016

21. Souweidane MM, Johnson JH Jr, Lis E: Volumetric reduction of a choroid plexus carcinoma using preoperative chemotherapy. J Neurooncol 43:167-171, 1999

22. Squire SE, Chan MD, Marcus KJ: Atypical teratoid/ rhabdoid tumor: the controversy behind radiation therapy. J Neurooncol 81:97-111, 2007

23. St Clair SK, Humphreys RP, Pillay PK, Hoffman HJ, Blaser SI, Becker LE: Current management of choroid plexus carcinoma in children. Pediatr Neurosurg 17:225-233, 1991-1992

24. Sun MZ, Ivan ME, Clark AJ, Oh MC, Delance AR, Oh T, et al: Gross total resection improves overall survival in children with choroid plexus carcinoma. J Neurooncol 116:179-185, 2014

25. Van Poppel M, Klimo P Jr, Dewire M, Sanford RA, Boop F, Broniscer A, et al: Resection of infantile brain tumors after neoadjuvant chemotherapy: the St. Jude experience. J Neurosurg Pediatr 8:251-256, 2011

\section{Disclosures}

The authors report no conflict of interest concerning the materials or methods used in this study or the findings specified in this paper.

\section{Author Contributions}

Conception and design: Mutchnick, Thatikunta, Thompson, Moriarty. Acquisition of data: Thatikunta, Thompson. Analysis and interpretation of data: Thatikunta, Thompson. Drafting the article: Thatikunta, Elster, Thompson. Critically revising the article: all authors. Reviewed submitted version of manuscript: Mutchnick, Thatikunta, Elster, Thompson, Spalding, Moriarty. Approved the final version of the manuscript on behalf of all authors: Mutchnick Study supervision: Mutchnick, Elster, Thompson, Huang, Spalding.

\section{Correspondence}

Ian Mutchnick, Division of Pediatric Neurosurgery, Norton Neuroscience Institute and Norton Children's Hospital, 210 East Gray St., Ste. 1102, Louisville, KY 40202. email: ian.mutchnick@ nortonhealthcare.org. 